\title{
Le partenariat école-musée en éducation relative à l'environnement : analyse et perspectives
}

Cécile Fortin-Debart

\section{OpenEdition}

1 Journals

Édition électronique

URL : http://journals.openedition.org/ere/6576

DOI : $10.4000 /$ ere.6576

ISSN : 2561-2271

Éditeur

Centr'ERE

Référence électronique

Cécile Fortin-Debart, « Le partenariat école-musée en éducation relative à l'environnement : analyse et perspectives », Éducation relative à l'environnement [En ligne], Volume 3 | 2002, mis en ligne le 20 mars 2002, consulté le 16 avril 2021. URL : http://journals.openedition.org/ere/6576 ; DOI : https://doi.org/ 10.4000/ere.6576

Ce document a été généré automatiquement le 16 avril 2021. 


\title{
Le partenariat école-musée en éducation relative à l'environnement : analyse et perspectives
}

\author{
Cécile Fortin-Debart
}

1 Depuis les années 1990, plusieurs circulaires ministérielles françaises incitent clairement les enseignants à utiliser les musées (Girault, 1999; Cohen, 2000). En effet, les musées de sciences sont des partenaires privilégiés de l'école, notamment parce que le ministère de l'Éducation nationale est, comme le souligne Eidelman (1998), un Ministère bis de la Culture avec ses 156 musées placés sous sa tutelle. De nombreuses directives officielles ont ainsi peu à peu cadré les sorties extra-scolaires que constituent les visites au musée.

De plus, « la préoccupation d'améliorer les rapports entre ces structures muséales et le monde scolaire semble être partagée au plus haut niveau de responsabilité puisque récemment deux ministres de l'Éducation nationale ont commandité des rapports d'étude à ce sujet » (Girault, 1999 ; voir aussi Héritier-Augé, 1991 et Geyssant, 1999). De nombreux travaux ont ainsi éclairé les relations entre l'école et le musée. Citons Caillet (1995), Girault et Guichard (1995), Jacobi et Coppey (1996), Cohen (2000), Girault et coll. (2000), etc.

3 Dans le cadre plus particulier de l'éducation relative à l'environnement (ERE), de nombreuses directives officielles incitent l'école à se tourner vers les musées. Parallèlement, le Bulletin Officiel de l'Éducation nationale du 11 mars 1993, invite les musées à développer l'accueil des classes dans un objectif d'ERE :

les musées d'histoire naturelle ont adopté depuis plusieurs années une politique d'ouverture vers le grand public qu'il faut encourager. Les services éducatifs dont disposent un bon nombre d'entre eux pourront dans certains cas être renforcés. Ils devront privilégier l'éducation à l'environnement tant au niveau de leurs productions (plaquette, valises, expositions, etc.) que de l'accueil des élèves qui devra faire une large place à l'aide aux projets. (B.O.E.N., 11/03/1993) 
Cette directive décrit plus particulièrement un programme éducatif mis en place avec la Villette (les Classes Villette Environnement) :

la Cité des Sciences et de l'Industrie (CSI) a ouvert un îlot sur ce thème. Il devrait permettre aux élèves et aux enseignants de prendre conscience de l'équilibre dynamique qui existe entre l'homme et son environnement et de la nécessité d'une gestion réfléchie des ressources de la planète. Le séjour à la CSI constitue le temps fort d'un projet qui peut s'étaler sur toute l'année scolaire. (B.O.E.N., 11/03/1993).

5 Nous allons voir qu'il existe une offre muséale organisée, qui propose aux scolaires des programmes éducatifs diversifiés. Ces programmes éducatifs sont en général réalisés en cohérence avec les programmes scolaires. De plus, dans certains musées, des enseignants détachés (c'est à dire des enseignants en poste qui intègrent quelques heures par semaine un service pédagogique de musée) participent à la création de ces programmes éducatifs.

6 Face à cette offre organisée, deux questions de recherche s'imposent. Une première question concerne l'adéquation sur le terrain avec les attentes des enseignants. La deuxième question, celle qui nous intéresse ici, est de définir de quelle manière cette offre peut devenir la base d'un véritable partenariat. Comment est organisée cette offre ? Comment les acteurs scolaires peuvent-ils l'utiliser ? Quels sont les présupposés pédagogiques que les enseignants doivent prendre en compte ? Quelles en sont les limites? Quelles sont les spécificités du musée que les enseignants peuvent exploiter et dans quel but? Quelles sont les pistes à privilégier pour un tel partenariat?

7 Nous proposons donc en premier lieu d'analyser cette offre muséale en élaborant une classification des différentes médiations proposées par les musées. Compte tenu des résultats obtenus par cette première analyse, nous soulignerons la nécessité dans le cadre d'un partenariat école-musée de former les enseignants d'une part et les responsables de musées d'autre part. Dans un troisième temps, nous nous demanderons si les stratégies pédagogiques développées par les musées de sciences intègrent les nouvelles orientations éducatives récemment identifiées par différentes recherches en ERE et en sciences de l'éducation. Ce travail présente donc un décryptage de l'offre muséale, afin que l'école et le musée s'associent au mieux pour le développement de l'éducation relative à l'environnement.

\section{Analyse de l'offre muséale}

8 Quelle est donc la nature de l'offre muséale et des différents programmes éducatifs destinés aux enseignants dans le domaine de l'environnement ? S'il existe des travaux de référence en éducation à l'environnement (Giordan et Souchon, 1992 ; Giolitto et Clary, 1994 ; Sauvé, 1997 ; etc.) et en muséologie de l'environnement (Davallon et coll., 1992), l'offre réelle des musées reste peu étudiée, tout en étant riche d'expériences sur le terrain. Nous proposons dans un premier temps de rappeler une typologie de cette offre que nous avons réalisée (Fortin-Debart, 1999).

9 Afin de construire notre typologie de l'offre muséale, nous avions mis en place une enquête auprès des différentes institutions muséales françaises partenaires possibles en contactant les responsables de ces institutions. Nous avons analysé parmi les différentes institutions muséales définies comme telles par l'ICOM (International Council of Museum) les écomusées (ECO), les parcs naturels régionaux (PNR), les parcs nationaux (PN), les musées d'histoire naturelle (MHN) et les centres de culture 
scientifique, technique et industrielle (CCSTI). Plus précisément, nous avons retenu les institutions muséales susceptibles de proposer une médiation environnementale. Nous avons alors envoyé à ces institutions un questionnaire et une demande de documentation sur leurs activités. Parmi les institutions muséales ayant répondu, 58 institutions ont fourni des données exploitables, représentant en moyenne $44 \%$ des institutions contactées. Notre analyse repose sur l'exploitation des réponses aux questionnaires et des descriptifs proposés par les musées (dossier pédagogique, catalogue d'exposition, etc.). Nous présentons ces données entre guillemets dans l'analyse suivante.

10 Nous avons utilisé comme grille d'analyse la typologie proposée par Boillot-Grenon (1999) qui a l'avantage de proposer une approche catégorielle simplificatrice et théorique des différentes conceptions et stratégies éducatives qui existent dans le domaine de l'ERE. Nous rappelons ici brièvement cette typologie, basée sur les différentes représentations de l'environnement. La représentation objective et biocentrique est axée sur la nature au sens large. La représentation technocentrique, sécuritaire et récursive s'appuie à l'origine sur la nécessité actuelle d'intervenir sur des systèmes artificiels qui sont à la fois naturels et sociaux. À l'usage, cette représentation s'est centrée sur les problèmes, les dysfonctionnements, les risques. Enfin, la représentation subjective et anthropocentrique est centrée sur le sujet humain et sa relation avec le milieu qui l'entoure : aménagement, usage, relations symboliques, etc.

11 En reprenant cette classification, nous rappelons donc notre propre typologie qui met en évidence d'une part les représentations dans lesquelles s'inscrivent les musées, et d'autre part, les différents types de programmes éducatifs qui en découlent. De cette première analyse, nous dégagerons des éléments essentiels à prendre en compte pour construire un véritable partenariat.

\section{L'approche biocentrique}

12 Un premier groupe d'institutions muséales (principalement des musées d'histoire naturelle et des parcs nationaux) s'inscrit dans l'approche biocentrique et écocentrique en abordant des thèmes relatifs aux êtres vivants (animaux et végétaux) et leurs milieux :

MHN 10 : Nous abordons la richesse des milieux, la diversité des êtres vivants, les difficultés qu'ils rencontrent suite aux actions humaines.

13 En abordant le thème de la richesse floristique et faunistique des milieux, ces musées s'orientent vers la notion de biodiversité, traitée souvent par le biais de l'écologie scientifique comme le prouve l'utilisation des termes suivants qui y font référence: milieux, équilibre, relation sol-végétation, fonctionnement du milieu naturel. De plus, les activités destinées aux scolaires font souvent appel à des disciplines de sciences naturelles telles que l'anatomie comparée ou la systématique. L'approche est donc fortement disciplinaire, centrée sur des approches naturalistes.

14 L'étude du patrimoine naturel s'applique en général à un niveau local : ces institutions muséales présentent ainsi la biodiversité spécifique de la région dans laquelle elles sont implantées. Les objectifs recherchés sont alors de connaître cet environnement local pour le respecter et le protéger. Dans cette perspective, le public scolaire apparait comme l'un des publics privilégiés : initier les adultes de demain à la connaissance et à la protection de leur milieu naturel et culturel est une des priorités du parc. Depuis 1982, le parc 
poursuit un programme d'actions pédagogiques de sensibilisation au milieu naturel en associant les personnels du parc et les enseignants (PN6).

Pour atteindre ces objectifs, les musées d'histoire naturelle présentent des collections naturalisées et offrent aux groupes scolaires la possibilité de visites libres (gérées par l'enseignant) ou de visites guidées avec un animateur. Les musées proposent également aux groupes scolaires des activités pédagogiques autour de ces collections : ateliers de découvertes, spectacles, animations, etc. Certains musées offrent plus qu'une simple consommation de leurs produits éducatifs et mettent en place un véritable partenariat avec des classes locales. Par exemple, lors de l'exposition «Le loup et l'homme » au MHN 9, certains enseignants ont basé leur préparation sur des dossiers pédagogiques, sur des pré-visites et des contacts établis avec les animateurs. Après la visite, plusieurs projets ont été organisés : travaux pluridisciplinaires, restitution de l'animation ou réalisation de livrets destinés aux autres classes de l'école, etc.

Quant aux parcs nationaux, leurs animations pédagogiques consistent le plus souvent en une découverte du patrimoine naturel, considéré comme une collection vivante. Il s'agit comme l'explique le PN 2 d'une expérience sensible au sein de la nature comme par exemple la découverte du milieu montagnard avec des gardes moniteurs dans le cadre d'un travail pédagogique sur le parc. Comme pour les muséums, l'exploitation par des scolaires des offres pédagogiques du parc peut devenir sous l'impulsion de certains enseignants et responsables pédagogiques de véritables partenariats.

L'analyse ainsi faite de ce premier groupe d'institutions muséales met en évidence une médiation axée sur le thème de la biodiversité, qui prend en compte la relation de l'élève avec la nature, dans une perspective locale et une approche scientifique. Sur cette base commune, la médiation dans un parc naturel est bien entendu très différente de celle proposée par un musée d'histoire naturelle: l'immersion dans un milieu naturel et l'observation de collections naturalisées aboutit à des apprentissages différents et surtout à des visions différentes de la nature. Cela suppose comme nous le verrons plus loin que les enseignants aient une certaine connaissance de ces présupposés pédagogiques.

\section{L'approche technocentrique}

18 Le deuxième type de médiation existe essentiellement dans les institutions muséales qui abordent l'environnement par le biais de relations réciproques entre l'homme et son milieu, et le plus souvent à travers certains problèmes environnementaux. Il s'agit en majorité des Centres de Culture Scientifique, Technique et Industrielle (CCSTI) même si quelques musées d'histoire naturelle y figurent. En général, ce sont des ensembles de problèmes environnementaux qui y sont exposés. Dans le CCSTI 6: l'exposition s'articule autour de quatre thèmes: l'énergie, l'air, les déchets et le bruit, à travers l'évocation des problèmes planétaires et la recherche des solutions à venir. La dimension de l'environnement est alors globale, à travers les thèmes récurrents de l'eau, des déchets ou encore du climat.

19 L'environnement étant traité sous forme de problèmes, ces institutions muséales invitent les élèves à participer à leur résolution en adoptant des comportements responsables. Le plus souvent cela correspond à participer au recyclage des déchets : cette exposition vise à responsabiliser chacun face aux déchets produits quotidiennement et insiste sur la nécessité du recyclage (CCSTI 7) ou à devenir des consommateurs 
responsables comme l'indique l'objectif du MHN 5: éduquer le citoyen à travers sa consommation d'eau quotidienne.

En terme de partenariat, au-delà de la simple visite guidée, c'est principalement à travers la pédagogie de projet que les musées cherchent à développer la participation et la responsabilisation des élèves, notamment par le biais des PAE (Projet d'Action Éducative) ${ }^{1}$. À titre d'illustration, le CCSTI 6 propose un exemple abouti de partenariat école-musée avec la production de plusieurs PAE lors de la présentation de l'exposition "Planète précieuse»: les PAE «Énergie solaire», PAE «La jette société», PAE "Énergie ", etc. reprennent ainsi des thèmes de l'exposition. La réussite de cette opération vient notamment de la mise à disposition des enseignants de nombreux moyens : tout d'abord l'Académie était un des partenaires permanents de l'itinérance régionale et a diffusé l'information en milieu scolaire. De plus, le CCSTI organisait des pré-visites permettant aux enseignants de cerner les thèmes traités et la manière dont ils pourront les exploiter avec leurs élèves ainsi que de préparer la visite afin qu'elle soit plus profitable. Un dossier enseignant a permis également d'approfondir en classe les thèmes de l'exposition après la visite. Enfin, ce CCSTI a organisé deux concours pour les scolaires sous forme de questionnaire afin de vérifier et d'approfondir les acquis. Cet exemple montre que l'organisation d'une pédagogie de projet dans le cadre d'un partenariat école-musée est un dispositif lourd à mettre en place, qui nécessite du temps et de l'organisation, mais susceptible de viser des vrais changements de comportements sur du long terme. Abordons à présent le troisième type de médiation proposé par les musées de sciences.

\section{L'approche anthropocentrique}

21 Certaines institutions muséales définissent l'environnement par rapport à l'Homme et aux systèmes sociaux, et s'inscrivent donc dans une vision anthropocentrique (BoillotGrenon, 1999). Cette manière d'aborder l'environnement est propre aux écomusées et aux parcs naturels régionaux. Les écomusées présentent cet environnement sociétal à travers l'évocation d'activités humaines caractéristiques d'un territoire ou d'une communauté : nous considérons l'environnement au sens le plus large possible: il s'agit pour nous de présenter l'essentiel des activités humaines passées ou présentes dans les domaines sociaux, historiques, géographiques, politiques, etc. (ECO 8). De même, les parcs régionaux abordent l'environnement humain qui est propre au territoire du parc: nos expositions traitent de la fragilité et de la richesse de l'environnement de l'homme dans le parc: écosystèmes, paysages, architecture, savoir-faire, culture locale (PNR 6). Le musée devient ici un acteur local, car il invite les habitants à participer à la gestion du parc (notamment avec les mesures agroenvironnementales destinées aux agriculteurs qui s'inscrivent dans la démarche d'entretien des paysages par exemple).

L'environnement est traité de manière fortement locale, cherchant à faire jaillir chez les élèves un sentiment d'identité et d'appartenance à un environnement spécifique, ayant sa propre histoire: par exemple, l'écomusée 8 souhaite faire découvrir ou redécouvrir l'identité d'un territoire à travers son histoire, son paysage, son architecture, sa culture passée et présente. Il s'agit de créer un sentiment d'appropriation du territoire (ECO 16). L'objectif est donc de valoriser ce patrimoine varié, à la fois naturel et culturel.

23 Les enfants vivant sur le territoire du parc constituent donc un des publics privilégiés en tant que futurs acteurs de ce territoire : le public scolaire, et d'abord celui qui fréquente 
les écoles situées dans le territoire du parc, est évidemment prioritaire à nos yeux: c'est en effet aux enfants d'aujourd'hui que reviendra demain la responsabilité de gérer et de valoriser le patrimoine que nous aurons à leur transmettre (PNR 4).

Dans cette perspective, les activités pédagogiques proposées sont la plupart du temps effectuées sur le terrain et en liaison avec des classes locales. Par exemple, l'ECO 6 organise pour les classes du canton une initiation à la notion de patrimoine, et une découverte in situ avec le responsable pédagogique de l'écomusée. De plus, la lecture de paysage est une activité souvent proposée aux scolaires. Dans la perspective d'un partenariat plus abouti, la plupart de ces institutions muséales (plus de la moitié) proposent également des classes découvertes, classes patrimoine ou encore classes environnement. De plus quelques parcs naturels régionaux organisent des séjours par l'intermédiaire de CPIE (Centre Permanent d'Initiatives pour l'Environnement) présents dans le parc. À titre d'illustration, un CPIE en partenariat avec un Parc naturel régional, organise des classes découvertes sur le thème " Eau et énergie ", à travers une approche locale du territoire. Les objectifs et les modalités du séjour sont organisés selon les programmes scolaires, modulables selon les objectifs pédagogiques et les attentes des enseignants. Une préparation en classe par les enseignants précédent la classe-découverte, suivie dans certains cas par différents projets de restitution destinées aux autres classes ou aux parents.

Cette typologie met donc en évidence l'existence de trois dynamiques différentes dans la médiation environnementale proposée par les institutions muséales : une dynamique de préservation de la biodiversité, une dynamique de responsabilisation face à certains problèmes environnementaux et enfin une dynamique de valorisation d'un territoire. Cette diversité est une richesse, elle permet de répondre à la diversité des situations pédagogiques mises en place par les enseignants. Encore faut-il qu'il y ait, comme nous l'avons vu à travers quelques exemples, une base de concertation entre les enseignants et le musée afin de dégager des approches et des objectifs communs. Nous allons voir que le partenariat peut aller encore plus loin, au-delà de la rencontre d'un enseignant avec un musée, pour viser un véritable échange entre les deux institutions, muséales et scolaires.

\section{Le partenariat à la recherche d'un contrat didactique}

Le partenariat école-musée implique, en amont de l'exploitation du musée par l'école, une réelle connaissance de chacun des partenaires. Cette connaissance nous semble en effet indispensable pour que le contrat didactique entre l'école et le musée soit clarifié et permette la définition de stratégies pédagogiques possibles. Les acteurs scolaires doivent par exemple avoir conscience de l'existence d'une diversité de partenariats possibles selon les institutions muséales, diversité mise en évidence précédemment avec la typologie. Chaque type de médiation repose en effet sur des présupposés pédagogiques spécifiques que l'enseignant a besoin de connaître. Les acteurs muséaux ont également tout intérêt à connaître et prendre en compte, d'une part les attentes institutionnelles de l'école et les attentes plus pragmatiques des enseignants, et d'autre part, les différents enjeux, objectifs et stratégies clarifiés par les différentes recherches menées dans le champ de l'ERE. Ces deux nécessités reposent donc sur la formation des acteurs muséaux et scolaires, comme nous allons le préciser à présent. 


\section{Pour une formation des enseignants sur les présupposés pédagogiques de la médiation environnementale par les musées}

Au préalable, l'enseignant doit être capable, notamment avec l'aide de la typologie proposée précédemment, de choisir le type d'institution muséale qui soit cohérent avec son projet : un thème lié à la protection d'une espèce animale implique logiquement un partenariat avec un musée d'histoire naturelle ou un parc national. De plus, la connaissance de cette offre permet aux enseignants de gérer les aspects disciplinaires. Par exemple, les enseignants doivent prendre en compte que certains musées s'inscrivent dans une approche disciplinaire spécifique (les musées d'histoire naturelle) ou au contraire dans une approche interdisciplinaire de l'environnement, basée sur une contextualisation sociale, historique, et politique de l'environnement. Plus concrètement, nous allons voir en reprenant deux exemples de médiation muséale qu'ils reposent de manière très précise sur des présupposés pédagogiques spécifiques (Girault, 1992).

28 Le musée d'histoire naturelle appuie son discours de préservation du vivant sur des collections d'animaux naturalisés. L'approche est essentiellement basée sur l'observation de spécimens, parfois recontextualisés dans une trame narrative (Girault et Guichard, 2000). L'exposition présente ainsi une certaine vision de la nature, et non la nature elle-même. Par exemple, dans un diorama (c'est-à-dire la présentation de spécimens dans une reconstitution de leur milieu), ce n'est pas la nature qui est représentée, mais le concept de milieu. Si l'enseignant l'ignore en exploitant un diorama, il va alors rentrer dans une approche simpliste de la nature. Est-ce que l'élève appréhendera alors toute la complexité de l'écosystème ou bien ne verra-t-il pas qu'une représentation esthétique d'un morceau de nature ? En effet, un diorama, aussi pédagogique soit-il pour intégrer la notion de milieu peut cependant difficilement faire apparaître tous les phénomènes dynamiques. Finalement :

n'y a-t-il pas un risque que le musée ne produise chez le visiteur un certain constat d'ignorance - ce qui en soi n'est pas une mauvaise chose, sauf à le rabattre sur ce qui est le plus apparent parce que visible et sensible (la présentation des « choses de la nature») et à le convier au bout du compte une simple pratique de consommation d'une image de la nature ? (Davallon et coll., 1992)

Toujours en ce qui concerne les muséums d'histoire naturelle, il serait intéressant d'envisager une formation des enseignants sur l'histoire des collections, afin qu'ils puissent réagir face aux questionnements des élèves. En effet, la présentation de collections naturalisées ne va-t-elle pas inciter les élèves à effectuer des prélèvements dans la nature? Comment expliquer à un élève que ce peut être en effet nuisible de collecter des objets de la nature quand c'était finalement la démarche première des musées d'histoire naturelle? Comment réagir face aux enfants qui s'indignent devant ces animaux tués à une certaine époque pour compléter les collections ? Comment finalement aborder la notion de vivant avec des animaux morts? Ce n'est qu'à travers une formation sur l'histoire des collections que des réponses peuvent être apportées à ce genre de questions (Cohen, 2000).

Dans un autre type de médiation, certains présupposés sont à prendre en compte pour éviter la construction d'une image défavorable des questions environnementales. Ainsi, pour les CCSTI, nous avons vu dans la typologie que la médiation était essentiellement basée sur la présentation de problèmes environnementaux. Il est important pour les 
enseignants de connaître cette approche, notamment parce qu'elle peut s'avérer délicate avec des enfants particulièrement jeunes. En effet :

la nécessité d'identifier des problèmes qui peuvent être définis et résolus par les enfants n'est pas facile considérant la complexité de nombreux enjeux environnementaux. (...) Des problèmes non résolus peuvent être source d'effroi. Finalement, je crois que la plupart sinon tous les problèmes du monde naturel ne devraient pas être transférés aux écoles dans l'espoir que les enfants les résolvent maintenant ou plus tard lorsqu'ils seront adultes. Peut-être devrions-nous prendre nos responsabilités comme adultes et le faire nous-mêmes. (Berryman, 1997, p. 5)

31 Le regard catastrophiste porté sur certains problèmes est susceptible alors de construire des sentiments de peur, mêlés à des sentiments d'impuissance peu favorables à une compréhension raisonnée des questions environnementales :

lorsque la problématique environnementale entre à l'école avec cette image catastrophiste, il devient difficile de faire appel aux possibilités de solution, de montrer que des systèmes bien gérés, en fonction de leurs propres dynamiques, peuvent connaître une évolution positive et constituer un modèle au niveau éducatif. (Torres Carrasco, 1998-1999)

Il apparaît ainsi nécessaire que l'enseignant ait conscience des limites d'une telle approche.

Il est donc important pour les enseignants d'être formé à un moment donné sur l'offre muséale. Dans quelle vision de l'environnement s'inscrit-elle ? Dans quelle approche disciplinaire ? Quels sont les objectifs ? Cet ensemble de présupposés pédagogiques ne sont pas forcément acquis pour la plupart des enseignants. Mais, en allant plus loin, nous pouvons nous demander si des enseignants qui n'ont jamais eu d'enseignement en ERE ont conscience de la diversité des visions, objectifs et approches qui caractérisent l'ERE en général. Ainsi, une formation des enseignants sur la diversité de l'offre muséale pourrait permettre de développer deux objectifs : le premier concernant la connaissance de l'offre muséale pour une exploitation des musées, le deuxième, et certainement le plus porteur, consistant en une prise de conscience de la diversité des approches en ERE mise en évidence à travers la diversité de l'offre muséale.

Dans cette perspective, un partenariat avec les IUFM (formation initiale et continue des enseignants) pourrait être envisagé. De plus, certains musées, qui ont développé des recherches sur la muséologie de l'environnement, pourraient proposer des sessions de formation continue destinées aux enseignants sur les questions environnementales. En effet, vu l'ampleur de la tâche éducative des enseignants, et vu la nécessité d'une formation sans cesse renouvelée face à une science qui progresse à grands pas et pénètre de plus en plus la société, l'ensemble de la communauté éducative dont font partie les musées se doit de contribuer aux processus de formation continue. Nous allons voir à présent que l'élaboration d'un contrat didactique entre le système scolaire et les musées passent également par une formation des acteurs muséaux.

\section{Pour une formation des acteurs muséaux en ERE}

Tout d'abord, nous pensons qu'il est important pour les musées de connaître les attentes institutionnelles de l'école. Il est par exemple indispensable de connaître les thèmes et les objectifs relatifs à l'ERE exigés par les programmes scolaires, afin de proposer une offre appropriée. En effet, lors d'une enquête, 79,3\% des enseignants interrogés souhaitaient des expositions et des activités en rapport direct ou 
complémentaire avec le programme scolaire (Quagliozzi et Cohen, 2000). Les musées devraient dès lors connaître l'ensemble des programmes scolaires, ainsi que leur évolution.

Dans un deuxième temps, nous pensons qu'il serait souhaitable de savoir ce qu'attendent de manière très pragmatique les enseignants lorsqu'ils emmènent leurs élèves au musée. Par exemple, dans le domaine de l'ERE pour lequel peu d'enseignants sont formés, que viennent-ils chercher au musée : Du contenu ? Une pédagogie ? Des enquêtes préalables destinées aux enseignants en formation ou en activité pourraient par exemple compléter des enquêtes sommatives plus habituelles, qui évaluent la satisfaction des enseignants ayant organisé une visite dans une perspective d'ERE.

Enfin, nous pensons que les personnels de musée ont chacun des cursus universitaires différents, et nombreux sont ceux qui n'ont jamais été formés à l'ERE. Des séminaires de travail pourraient être ainsi organisés: ils regrouperaient les personnels des services pédagogiques, mais aussi ceux qui conçoivent les expositions, pour une formation par des chercheurs en ERE. À titre d'illustration, nous pouvons citer deux exemples de séminaires: l'un organisé au Muséum national d'Histoire naturelle de Paris offrait aux personnels du service d'action pédagogique et culturelle une aprèsmidi de formation à l'ERE. L'autre dépasse notre cadre d'études du partenariat écolemusée en France, car il a eu lieu au Québec, à l'Insectarium de Montréal (mai 2001), mais fut l'occasion d'une expérience enrichissante: des responsables de musées montréalais (Biodôme, Insectarium, Planétarium, Biosphère) ont rencontré pour une journée d'échanges des chercheurs et des étudiants issus des domaines de l'ERE et du partenariat école-musée. Cette journée de travail fut également l'occasion d'un échange entre expériences québécoises et françaises.

Ce genre d'initiatives nous paraît intéressant pour que les acteurs muséaux prennent conscience des caractéristiques de l'ERE. En effet, la connaissance d'un certain nombre de points en ERE peut permettre aux expositions ou animations d'aborder au mieux les questions environnementales. Par exemple, il peut être important pour des musées de sciences de prendre conscience de la nécessité de resituer une question environnementale dans son contexte politique, économique et social, et ce afin que la science n'apparaisse comme la seule solution, alors que ces problèmes soulèvent " pourtant bien des questions sur les plans politiques, sociaux et éthiques, enjeux qui ne relèvent donc pas uniquement de l'expertise scientifique » (Bader, 1998-1999).

De plus, nous pensons qu'il est important que les musées, lorsqu'ils abordent une question environnementale, trouvent un équilibre entre responsabilité individuelle et responsabilité collective. En effet dans certaines expositions, "c'est souvent le comportement individuel qui est visé, plus que les conditions sociales, économiques ou politiques, réduisant d'autant le risque de controverses " (Davallon et coll., 1992). Par exemple, de nombreuses expositions commencent avec l'évocation de problèmes environnementaux globaux: la pollution atmosphérique, les déchets industriels et radioactifs, etc. pour finir en général sur un objectif de participation au tri sélectif et au recyclage. L'équilibre entre individuel et collectif n'est pas respecté et peut aboutir à un sentiment de découragement chez l'apprenant à qui on présente rarement d'autres alternatives plus collectives et globales.

Une formation appropriée sur les questions environnementales, et les rapports individus/société dans ces problématiques apparaît ainsi nécessaire. De même, il serait intéressant selon nous de former les acteurs museaux sur certaines dérives 
couramment rencontrées. Par exemple, nous avons vu dans la typologie que les écomusées avaient tendance à présenter souvent le territoire tel qu'il était dans le passé. Si une perspective historique est toujours intéressante pour comprendre l'aménagement d'un territoire, certains écomusées proposent parfois une image trop idyllique de ce passé, empreint d'une certaine nostalgie. Or si les questions environnementales nécessitent souvent de regarder en arrière, elles souffrent également de ces images idylliques du passé, associées à des images négatives du progrès.

41 Autant que les enseignants, les acteurs muséaux ont besoin selon nous d'une formation à l'ERE. Il serait aussi intéressant que les trois univers de l'école, des musées et de la recherche en ERE se rencontrent davantage pour des échanges. Comme nous l'avons déjà évoqué, est-ce qu'une double formation ne pourrait être envisagée : Une formation par et pour le musée ? Une formation sur les questions environnementales par le musée qui déboucherait naturellement sur une formation à l'exploitation du musée. Nous allons voir que l'exploitation de musée peut également orienter cette formation vers une nouvelle approche éducative des questions environnementales.

\section{Une nouvelle approche éducative de l'ERE dans les musées de sciences}

Des recherches récentes en ERE (présentées notamment dans le volume 1 de la revue Éducation relative à l'environnement: Regards, Recherches, Réflexions) ont montré la nécessité d'orienter l'ERE vers la réflexivité, basée sur «des capacités de réflexion permettant de clarifier les valeurs sous-jacentes à tout choix de stratégie environnementale » (Croizer et Goffin, 1998-1999).

Cette perspective nous semble être une voie de recherche indispensable pour répondre aux nouveaux enjeux de l'ERE, qui se doit de dépasser le stade de la sensibilisation pour inviter chaque personne à rentrer dans un processus réflexif de compréhension des réalités environnementales, en vue d'un agir collectif et individuel responsable. Si l'on se réfère aux différentes stratégies et modèles proposés en ERE (Sauvé, 1997), il apparaît que l'approche réflexive et critique, l'analyse et la clarification des valeurs, des représentations reposent essentiellement sur le débat et la discussion. Dans cette perspective se pose alors la question suivante : le musée peut-il être un lieu de débat et donc un partenaire pour une ERE réflexive?

Dans la revue de la bibliographie relative à la muséologie, plusieurs études montrent comment le musée peut être potentiellement un lieu de débat et de discussion. Par exemple, de nombreux travaux issus de recherches appliquées aux évaluations d'expositions ont mis en évidence que la plupart des visites au musée se font en groupe, et qu'il existe à l'intérieur des groupes des interactions sociales, des phénomènes relationnels qui constituent une forme d'«apprentissage mutuel» (Cordier, 1994). Ces évaluations ont montré que la conversation est éventuellement, et selon les circonstances, au cœur de cet apprentissage. Elle n'est pas obligatoirement présente, mais elle est possible :

Hensel s'est aperçue qu'au musée les conversations commencent et s'interrompent sans arrêt, et qu'au sein du groupe elles permettent d'attirer l'attention des autres en direction de l'objet. Les conversations qui se déroulent face à un élément de 
l'exposition participent d'une conversation plus globale qui se poursuit tout au long de la visite. (Dierking, 1995) un lieu de débats pour des questions environnementales et qu'il offre donc la possibilité aux enseignants de s'orienter vers de nouvelles approches éducatives en ERE. Les objectifs sont multiples. D'abord, comme nous l'avons vu, il s'agit de répondre aux nouveaux enjeux de l'ERE souhaitée de plus en plus réflexive. Il s'agit également de répondre à une certaine demande des enseignants qui face aux débats et aux controverses, «se plaignent de l'absence de ressource et de leur manque de formation " (Delalande-Simonneaux, 2000).

50 répond à une volonté institutionnelle d'intégrer le débat à l'école. Par exemple, en Éducation Civique, le débat permet de répondre à divers objectifs : «les débats qui concernent la gestion des déchets radioactifs et la recherche biologique et médicale 
permettent de comprendre l'enjeu démocratique que représente l'information des citoyens et la prise de conscience de leurs responsabilités " (programme d'éducation civique, 3ème, Éducation nationale, 1999). De même, pour l'ensemble des disciplines, il est conseillé aux enseignants de contextualiser leur enseignement dans une approche sociale du savoir, qui passe souvent par les controverses, les débats. Ainsi, dans une approche disciplinaire, il s'agirait de privilégier une démarche épistémologique qui rapproche la connaissance «de ses racines idéologiques ou métaphysiques, de son histoire, de ses controverses, du champ de force dans lequel elle est engendrée, vérifiée, affirmée ou combattue, des valeurs et intérêts qu'elle affaiblit ou renforce dans la société » (Perrenoud, 1998).

51 Ainsi, que ce soit dans le cadre d'une discipline nouvelle (l'éducation civique) ou dans le cadre disciplinaire d'un rapport épistémologique au savoir, le débat apparaît aujourd'hui comme une stratégie importante dans l'enseignement scolaire: «un citoyen est celui qui peut parler, qui peut s'exprimer, qui peut aller sur le forum, et qui est capable d'exposer son point de vue. Nous pourrions exiger de l'école qu'elle forme plus les élèves à cet accès à la parole » (Meirieu, 1998).

L'exploitation d'une stratégie basée sur le débat et proposée par un musée de sciences est donc une orientation intéressante pour le partenariat école-musée. Entre autres, le musée pourrait être envisagé comme un lieu de formation des enseignants aux débats et controverses scientifiques. De plus, les classes pourraient exploiter le musée dans cette perspective de débat: la visite peut soit susciter l'intérêt ou le questionnement avant un débat en classe, soit être l'occasion d'un débat au sein du musée. Même si l'enseignant souhaite organiser un débat dans la classe, le rôle d'un médiateur (muséal ou autres) est important. En effet, la présence d'une personne autre que le professeur brise le rapport professeur-élèves qui pourrait dénaturer le débat. En effet, les élèves essaient de formuler des opinions qui satisferont leur enseignant: «ils sont influencés par le rapport institutionnel établi avec l'enseignant. Ils cherchent plus ou moins consciemment à percer les opinions des enseignants et ont tendance à moins exprimer leur argumentation s'ils se sentent en désaccord avec l'enseignant» (DelalandeSimonneaux, 2000). Cependant l'enseignant peut être présent, car il est intéressant pour lui "de voir les élèves sous un angle différent où la personnalité transparaît " (Delalande-Simonneaux, 2000).

Malgré ces potentialités mises en évidence, le débat au sein du musée rencontre de nombreux obstacles et certaines limites. Par exemple, il est évident que d'autres lieux (les cafés-sciences, cafés-citoyens dans des cafés en ville) offrent une convivialité plus propice aux débats que le musée, «lieu sacré du savoir ». Soulignons également les initiatives du café-sciences de Lyon qui organise dans les lycées des débats, autour de cafés, en dehors des heures de classes. Nous avons également mis en évidence une autre difficulté à travers l'exemple du Muséum national d'Histoire naturelle de Paris qui donne rarement accès à une information plurielle. En effet, dans les expositions, «les diverses théories scientifiques, les conflits de représentations qui sont en jeu » sont rarement formulés (Girault et Fortin-Debart, 2002). En fait, aux difficultés de muséographier le débat, s'ajoutent des réticences institutionnelles, par exemple avec la peur de voir l'institution muséale "se transformer en un lieu de communication militante » (Girault et Fortin-Debart, 2002). Dans le cadre d'un partenariat école-musée, il est donc important de prendre en compte non seulement les potentialités du musée, mais aussi ses limites, afin d'ouvrir le questionnement et les perspectives. Des 
recherches plus approfondies devraient permettre de préciser cette nouvelle voie de la médiation muséale, qui invite les élèves et les enseignants dans une nouvelle approche des questions environnementales.

\section{Conclusion}

La typologie proposée en première partie souligne la diversité de l'offre muséale dans le domaine de l'environnement. Chaque type de médiation repose sur des présupposés pédagogiques différents qui impliquent une formation des enseignants à l'exploitation du musée, qui pourrait être ainsi l'occasion d'une formation aux questions environnementales. Par ailleurs, il apparaît qu'une formation des personnels de musée est nécessaire afin que la médiation muséale bénéficie des apports issus du domaine de l'ERE. Dans cette perspective, le partenariat école-musée, plus qu'une simple consommation organisée, sera un véritable outil d'ERE, reposant sur des échanges d'expertise entre école, musée et ERE. Par exemple, nous avons montré les potentialités et les limites du musée pour devenir un partenaire pour une ERE réflexive. Cette étude a donc mis en évidence toute la richesse du partenaire musée, à condition que ce partenariat repose d'une part sur une formation des différents partenaires, et d'autre part sur un cadre pédagogique rigoureux, qui implique des recherches et des échanges entre les différents domaines : enseignement scolaire, médiation muséale et ERE.

\section{BIBLIOGRAPHIE}

Arpin, R. (1997). Des musées pour aujourd'hui. Québec : Éditions Multimondes, Musée de la Civilisation.

Bader, B. (1998-1999). Enseignement scientifique, technologique et éducation relative à

l'environnement sans recul réflexif : une conjugaison risquée. Éducation relative à l'environnement : Regards, Recherches, Réflexions, 1, 187-194.

Berryman, T. (1997). Bulletin d'information du Centre de la Montagne, $n^{\circ} 13$. Montréal : Centre de la Montagne.

Boillot-Grenon, F. (1999). Clarifier les représentations des partenaires de l'éducation relative à l'environnement. Aster, $\mathrm{n}^{\circ}$ 29, 61-84.

Bradburne, J.M. (1998). Problématique d'une création : NewMetropolis. In B. Schiele et E.H. Koster (dir.), La révolution de la muséologie des sciences. Québec et Lyon : Éditions Multimondes et Presses Universitaires de Lyon. p. 39-77.

Caillet, E. (1995). À l'approche du musée, la médiation culturelle. Lyon : Presses Universitaires de Lyon.

Cohen, C. (2000). Contribution à l'étude des relations entre l'école et le musée. Vers une formation de l'enfant visiteur. Thèse de Doctorat, Muséum National d'Histoire Naturelle, Paris. 
Cordier, J.P. (1994). La transmission familiale au musée. Paris : CNRS/Université Descartes. Croizer, C. et Goffin, L. (1998-1999). Les relations entre éducation et formation en environnement : un champ de recherche. Éducation relative à l'environnement : Regards, Recherches, Réflexions, 1, 141-148. Davallon, J., Grandmont, G. et Schiele, B. (1992). L'environnement entre au musée. Lyon et Québec : Presses Universitaires de Lyon et Musée de la Civilisation.

Delalande-Simmoneaux, L. (2000). Didactique et éducation biotechnologique. Note de synthèse pour l'habilitation à diriger des recherches, Université de Rouen.

Dierking, L.D. (1995). Rôle de l'interaction sociale dans l'expérience muséale. Publics et Musées, $n^{\circ}$ 5, 19-43.

Eidelman, J. (1998). L'espace muséal scientifique et ses publics. La lettre de l'OCIM, n 55, 3-5. Fortin-Debart, C. (1999). Analyse de l'offre des institutions muséales en médiation environnementale. Aster, ${ }^{\circ}$ 29, 85-100.

Geyssant, J. (1999). Rapport d'étape au ministre d'État, Ministre de l'Éducation nationale, de la Recherche et de la Technologie sur la collaboration entre les établissements d'enseignement et les institutions muséales scientifiques. Non publié.

Giolitto, P. et Clary, M. (1994). Éduquer à l'environnement. Paris : Hachette.

Giordan, A. et Souchon, C. (1992). Une éducation pour l'environnement. Nice : Z’Éditions.

Girault, Y. (1999). L'école et ses partenaires scientifiques. Aster, nº 29, 3-8.

Girault, Y. (1992). Mieux les connaître pour mieux préparer la visite. Revue Spectre, 21 (5), 29-32. Girault, Y. et Fortin-Debart, C. (2002). Le musée forum, un difficile consensus. L'exemple du Muséum national d'Histoire naturelle. Revue Quaderni, sous presse.

Girault, Y. et Guichard, F. (2000). Spécificité de la didactique muséale en biologie. In J. Eidelman et M. Van Praët (dir.), La muséologie des sciences et ses publics. Paris : Presses Universitaires de France. p. 63-74.

Girault, Y. et Guichard, F. (1995). Problématique et enjeux du partenariat école/musée à la Grande Galerie de l'Évolution. Publics et Musées, n 7, 69-91.

Girault, Y. et coll. (2000). Prise en compte des intérêts des élèves dans le cadre de l'appropriation des savoirs scientifiques dans les espaces muséaux. Rapport de recherche. Paris : Comité National de Coordination de la Recherche en Éducation.

Habermas, J. (1992). L'espace public, trente ans après. Quaderni, nº 18. Habermas, J. (1962). L'espace public. Paris : Éditions Payot.

Héritier-Augé, F. (1991). Les musées de l'Éducation nationale. Mission d'étude et de réflexion. Rapport au Ministre d'État, Ministre de l'Éducation nationale. Paris : La documentation française

Jacobi, D. et Coppey, O. (1996). Musée et éducation : au-delà du consensus, la recherche du partenariat. Publics et Musées, $\mathrm{n}^{\circ}$ 7, 10-22.

Meirieu, P. (1998). Le nécessaire accès à la parole. Cahiers pédagogiques, supplément $\mathrm{n}^{\circ} 4$ : mL'éducation à la citoyenneté, 18.

Perrenoud, P. (1998). Le débat et la raison. Cahiers pédagogiques, supplément $n^{\circ} 4:$ L'éducation à la citoyenneté, 4-7.

Quagliozzi, A. et Cohen, C. (2000). Vers le partenariat école-musée : mobilisation des enseignants et expertise des élèves. In J. Eidelman et M. Van Praët (dir.), La muséologie des sciences et ses publics. Paris : Presses Universitaires de France. p. 281-289. 
Rasse, P. (1997). Les musées à la lumière de l'espace public. Des collections à la communication. Nice : Université Sophia Antipolis, Projet d'Habilitation à la Direction de Recherches.

Sauvé, L. (1997). Pour une éducation relative à l'environnement. Montréal : Éditions Guérin.

Torres Carrasco, M. (1998-1999). L'éducation relative à l'environnement en Colombie : un processus construit dans une perspective de recherche. Éducation relative à l'environnement : Regards, Recherches, Réflexions, 1, 97-117.

\section{NOTES}

1. Les PAE, créés en 1981, visent dans le premier et le second degré, à faire pratiquer aux élèves des activités interdisciplinaires en liaison avec la communauté éducative locale (parents, associations, collectivités locales, etc.), en mettant en œuvre une démarche de projet, et en prenant appui sur les compétences spécifiques d'intervenants extérieurs.

\section{RÉSUMÉS}

La plupart des musées de sciences abordent dans leurs expositions et, ou animations des thématiques environnementales et proposent aux scolaires divers programmes éducatifs. Le but de cet article est d'analyser cette offre muséale et de souligner la nécessité, pour développer un véritable contrat didactique entre les partenaires, de proposer aux enseignants et aux responsables pédagogiques de musées une formation spécifique. De plus, l'auteur s'interroge sur les potentialités des musées de sciences à développer une éducation relative à l'environnement réflexive, à travers la notion de débat au sein des expositions scientifiques.

Most science museums include in their exhibitions and/or animations, environmental themes and offer different education programs to schools. This article aims to analyze this museumrelated offer and underline the need for a specific training for teachers and people in charge of the animation and education department in the museums. Moreover, the author explores the potential of the science museums to develop a reflexive environ-mental education based on debates and discussions inside the exhibitions.

\section{AUTEUR}

\section{CÉCILE FORTIN-DEBART}

Cécile Fortin-Debart prépare actuellement une thèse de doctorat en muséologie des sciences naturelles et humaines au Muséum national d'Histoire naturelle (Paris, France). Elle est membre de l'Équipe de Recherche sur la Médiation Muséale, dirigée par le professeur Yves Girault. 